\title{
La pertinencia de la tesis en Ciencias Sociales
}

\author{
Teodoro Bustamante
}

\begin{abstract}
RESUMEN
En el ECUAdor, ha SURgido Un debate SObre la ORganización de la EDUCACión SUPERIOR (ARCOS, 2012; Villavicencio, 2013) que se inscribe en un debate mayor (De Sousa Santos, 2005). Como parte de él se ha PUESTO EN TELA DE JUICIO LA PERTINENCIA DE LA ELABORACIÓN DE LA TESIS COMO TRABAJO FINAL DE VARIAS CARRERAS Y DE DIFERENTES NIVELES DE FORMACIÓN. A TRAVÉS DE UN TRABAJO EXPLORATORIO HEMOS PROCEDIDO A ANALIZAR ALGUNOS VARIABLES CONCENTRADAS FUNDAMENTALMENTE EN LA BIBLIOGRAFÍA REFERENCIADA EN UN CONJUNTO de tesis de Ciencias Sociales con el propósito de entender mejor los aspectos de la Vida aCAdémica JUSTAMENTE a TRAVÉS DEL TRABAJO DEL TESISTA.
\end{abstract}

Palabras clave: Ciencias Sociales - tesis - Publicaciones.

\section{Abstract}

A discussion SURrounding the organisation of Higher EdUCATION has EMERged IN ECUAdor (ARCHES, 2012;) Villavicencio, 2013) as part of a larger debate (De Sousa Santos, 2005). One aspect of this debate has BEEN TO QUESTION THE RELEVANCE OF THE THESIS AS A FINAL WORK IN VARIOUS COURSES OF STUDY AND DIFFERENT LEVELS OF TRAINING. WE HAVE ANALYZED VARIABLES, CONCENTRATING PRIMARILY ON THE LITERATURE REFERENCED IN A SET OF SOCIAL SCIENCE THESES IN ORDER TO BETTER UNDERSTAND THE ASPECTS OF ACADEMIC LIFE PRECISELY THROUGH THE WORK OF THE STUDENT.

KEY WORdS: Social SCIENCE - THESIS - PUbliCATIONS. 
$\mathrm{E}$ ste trabajo explora las características de la bibliografía citada en un total de 72 tesis de Ciencias Sociales de dos instituciones de educación superior en el Ecuador. Se analizan temas tales como el tipo de documentos utilizados, los sitios donde han sido publicados, las nacionalidades de los autores, las editoriales usadas, los idiomas de los documentos, la antigüedad de los documentos a la fecha de su utilización, y el género de los autores citados y de los autores de las tesis.

Los 72 trabajos académicos se distribuyen en un período de tiempo de 37 años. La más antigua fue aprobada en el año 1977, y la última en el 2014. El análisis se complementó con una búsqueda de los datos más simples respecto a un número mayor de tesis según constan en las bases de datos de las bibliotecas de algunas instituciones de educación superior.

Las tesis revisadas consignan en sus bibliografías un total de 5.413 referencias bibliográficas que corresponden a 4.728 documentos elaborados por 3.231 autores distintos. ${ }^{2}$ Aunque esto nos habla más que de una manera muy parcial de la calidad misma del documento, nos puede ayudar a indagar sobre ciertas relaciones que contribuyan a una reflexión de lo que sucede en estos niveles académicos, y por lo tanto de ideas que pueden ser exploradas. En todo caso es también una aproximación que puede sustentar trabajos ulteriores de mayor detalle y sistematicidad.

Del universo de estudio encontramos un promedio de 75,18 referencias bibliográficas hechas en la tesis. Un número ligeramente mayor en las tesis de maestría: 77, mientras que en pre-grado la media es de 73.6. Esto nos está indicando algo sobre la amplitud de los recursos consignados usados en estos trabajos de graduación. Podemos construir un indicador de referencias por página y este es de 0,52 .

Como otra variable dentro del análisis de de la bibliografíam nos pregumtamos también ¿qué tipo de textos o fuentes se utilizan en estos trabajos? Esto lo podemos ver en el gráfico 1.

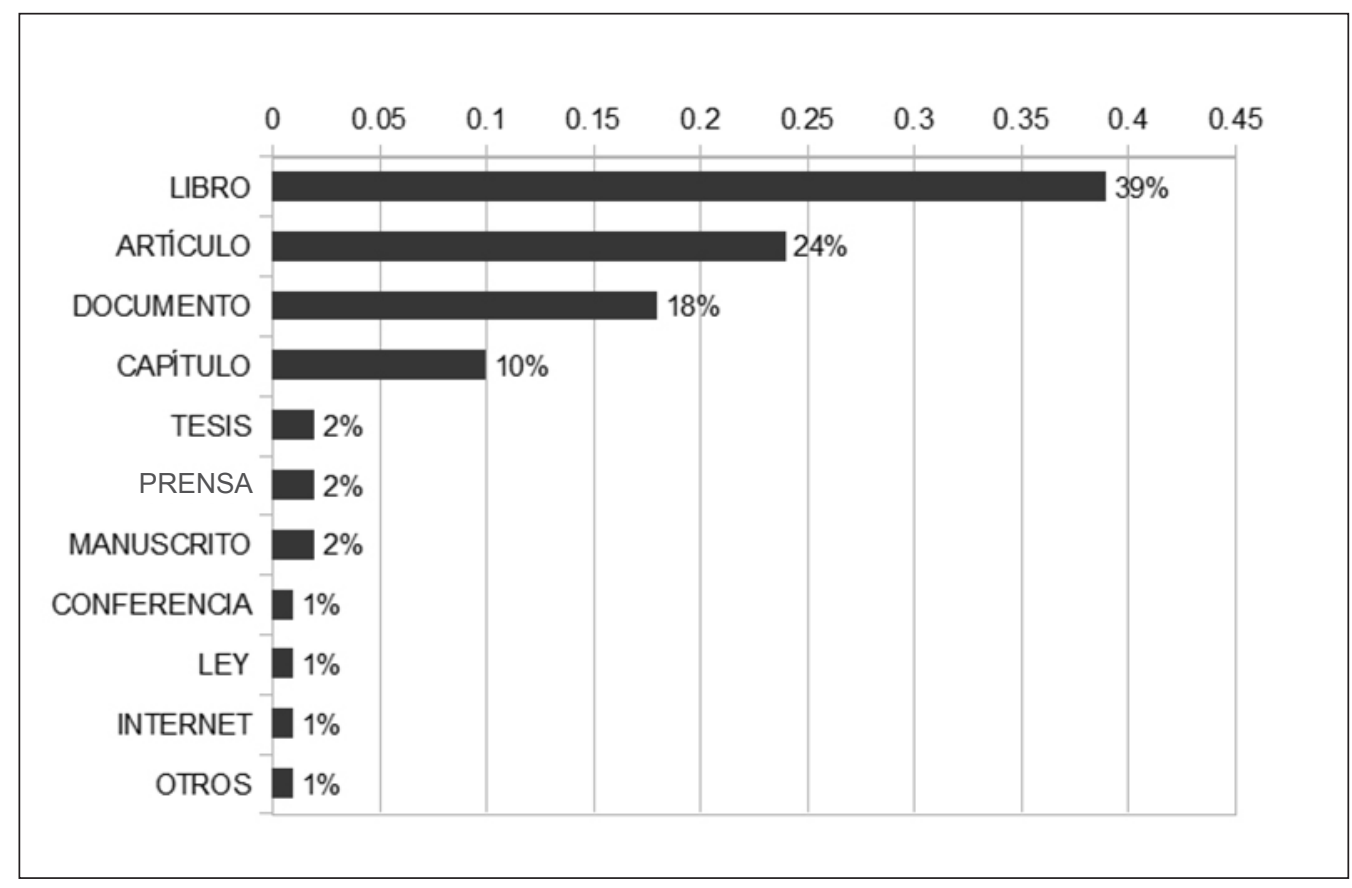

Gráfico 1. Tipo de documentos consignados en las bibliografías de las tesis. Elaboración propia.

2 Cuando hay actores colectivos, o más de un autor, estos han sido considerados como un autor adicional distinto de cada uno de las personas que puedan escribir individualmente. 
Estos datos nos muestran algunas cosas interesantes. La primera de ellas es la importancia que tienen los libros como material a ser utilizado. Proponemos que el libro es la fuente de mayor elaboración de las posibles y su alta participación nos estaría indicando que la tesis constituye un esfuerzo importante por actualizarse con materiales elaborados, es decir teóricos. El segundo rubro sería el de los artículos. Estos tienen también un fuerte aporte teórico y son elementos de importante actualización. Un tipo diferente y muy interesante de información es el que aportan los documentos. Estos son materiales muy diversos, en gran medida documentos oficiales y son a nuestro juicio un indicio del esfuerzo de investigación concreto sobre la realidad. Su participación porcentual es apreciable. Cómo siguiente rubro debemos señalar a los capítulos de libros. Esta es una forma de publicación en medios académicos, que si bien está siendo desplazada por el artículo en revistas constituye todavía una forma importante de publicación académica. Por último abordemos algunos de los rubros marginales. El internet, aparece con escaso peso, así como algunas otras fuentes de información. Esto puede tener una relación con la velocidad con la cual se incorporan nuevas tecnologías. ${ }^{3}$

Un comentario aparte merece la proporción de tesis utilizadas. El valor del 2,1 \% nos indica que el trabajo de las tesis sí es utilizado por otras personas en actividad académica. Este valor sin embargo es con seguridad una subestimación, pues si una tesis es luego objeto de una publicación, ya sea como fue presentada o con modificaciones, en este sistema será consignada como un libro. Permítasenos agregar que algunas tesis de grado y de pregrado han tenido importancia como textos apara las Ciencias Sociales. Señalemos por ahora sólo la de Fernando Velasco (1972).

Al analizar el lugar donde han sido publicadas las referencias bibliográficas mencionadas en la tesis, nos encontramos con una muy apreciable concentración en la ciudad de Quito (recordemos que todas son tesis elaboradas en esta ciudad). Esto podría llevarnos a pensar en un cierto parroquialismo en el mundo intelectual al cual acuden los tesistas, pero esto merecerá una discusión ulterior. Llaman la atención otros elementos, como por ejemplo: entre las diez primeras ciudades de publicación, todas son ciudades hispanohablantes; y el hecho de que la única otra ciudad del Ecuador que aparece con importancia como referencia bibliográfica es Guayaquil, y esta a penas con un modesto $2,16 \%$.

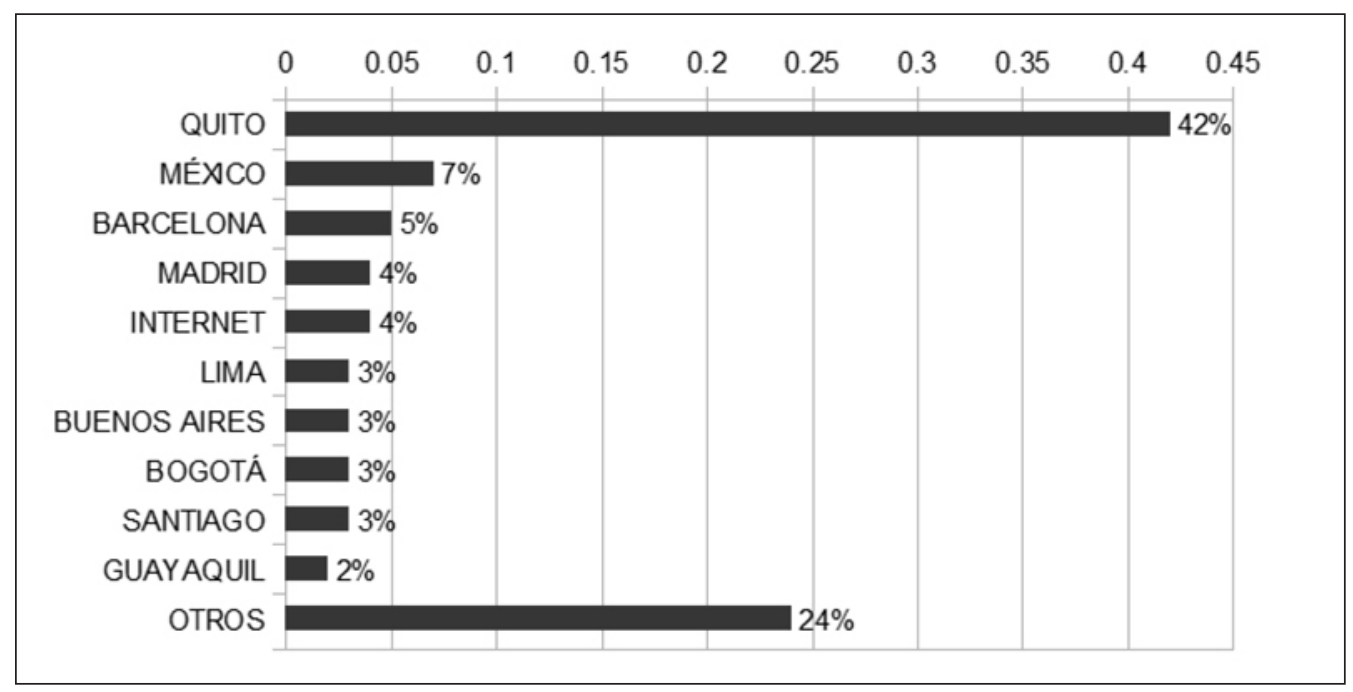

Gráfico 2. Ciudades de PUblicación de los documentos consignados En laS Bibliografías de las teSis REVISADAS. ELABORACIÓN PROPIA.

3 Debemos anotar sin embargo que como documento de internet aparece aquí solamente lo que ha sido publicado exclusivamente en internet, es decir no están incluidos ni textos publicados en papel y accedidos a través del internet ni artículos de revistas electrónicas que han sido considerados como artículos de revistas. 
En relación a la bibliografía, nos preguntarnos también cuáles son las editoriales más importantes en cuanto proveedoras de los materiales para la elaboración de la tesis. Aquí los resultados nos muestran una dispersión mucho mayor. En realidad hay 1700 editores distintos. Pero si analizamos las fuentes individuales nos topamos que el dato con mayor frecuencia de aparición en el registro de editorial es $\mathrm{SD}$, es decir, sin dato, que llega a representar un $8.87 \%$ de todas las referencias. Esto tiene muchas explicaciones posibles. Por una parte es cierto que con frecuencia existen documentos, especialmente los antiguos, en que este dato no se consigna. En otros casos no es pertinente como cuando se menciona una comunicación personal o documentos inéditos, pero estos representan una minoría. Tal vez este dato constituye un llamado de atención al rigor formal con el cual se elaborar las bibliografías académicas.

Como segundo porcentaje está la editorial Abya Yala que cumple un papel importante en cuanto proveedora de los documentos utilizados. Las dos siguientes fuentes de documentos son también ubicadas en territorio nacional, Una universidad y el registro oficial que nos indica en gran medida la incidencia que tiene en el trabajo académico la referencia a textos legales. Consta también otra entidad oficial como proveedora de información. Esto es el Banco Central del Ecuador.

\begin{tabular}{|l|l|}
\hline CuAdro 1. Editoriales MÁS FreCUENTEMENTE CitAdAs. Elaboración Propia. \\
\hline sd & $6,50 \%$ \\
\hline Abya Yala & $6,28 \%$ \\
\hline Flacso Ecuador & $4,93 \%$ \\
\hline Registro Oficial & $1,88 \%$ \\
\hline Siglo xxi & $1,81 \%$ \\
\hline Puce & $1,76 \%$ \\
\hline Fondo de Cultura Económica & $1,44 \%$ \\
\hline Paidos & $1,15 \%$ \\
\hline Internet & $1,02 \%$ \\
\hline caap & $0,96 \%$ \\
\hline Banco Central del Ecuador & $0,89 \%$ \\
\hline Corporación Editora Nacional & $0,78 \%$ \\
\hline Grijalbo & $0,61 \%$ \\
\hline Archivo Histórico del Guayas & $0,55 \%$ \\
\hline Instituto internacional de gobernabilitat de Catalunya & $0,54 \%$ \\
\hline Otras & $68,91 \%$ \\
\hline
\end{tabular}

Dado que en el gráfico tres el desglose ha sido efectuado a nivel individual no aparecen otros aspectos interesantes, y este es el que se refiere al tipo de entidades que son las editoras de estos materiales. Esto lo podemos ver el gráfico cuatro. En él aparece la importancia de las editoriales, de las universidades, que incluyen las editoriales universitarias. También aparecen en un papel muy importante las organizaciones no gubernamentales. Inclusive con más importancia que los organismos oficiales. Se puede constatar la importancia también de los organismos internacionales. ${ }^{4}$

4 En el caso de las revistas, si ha sido posible identificar si están editadas por organismos internacionales, por ONGs o por universidades, se ha registrado como tipo de entidad a esta última. Así por ejemplo las referencias a la revista Ecuador Debate, han sido consignada conjuntamente con los libros editados por el CAAP. Iconos, conjuntamente con los textos de la Flacso Ecuador, y la revista de la CEPAL como un organismo Internacional. 


\begin{tabular}{|l|c|}
\hline CUAdro 2. TiPo de entidades QUE PUblican los MATERIALES USAdOS EN LAS TESis. Elaboración Propia. \\
\hline EDITORIAL & $41,11 \%$ \\
\hline UNIVERSIDAD & $21,59 \%$ \\
\hline ONG & $16,93 \%$ \\
\hline OFICIAL & $13,44 \%$ \\
\hline REVISTA & $8,34 \%$ \\
\hline ORGANISMO INTERNACIONAL & $7,59 \%$ \\
\hline PERIODICO & $3,02 \%$ \\
\hline INSTITUCION & $2,99 \%$ \\
\hline EMPRESA & $2,58 \%$ \\
\hline INTERNET & $1,73 \%$ \\
\hline MANUSCRITO & $1,64 \%$ \\
\hline
\end{tabular}

Si bien ya tenemos algunos indicadores sobre cómo se organizan las influencias académicas, podemos experimentar con otra variable ¿Cuál es el origen nacional de los autores citados. Para ello hemos tabulado la nacionalidad de nacimiento de los autores y obtenemos el gráfico cinco. En el vemos que los autores ecuatorianos constituyen una parte importante de las referencias utilizadas. También observamos cuales son los países metropolitanos que más influencia tienen (EEUU, Francia y España) y cuáles son los países latinoamericanos con respecto a los cuales hay más interacción a este nivel (Perú, Colombia Argentina y Chile). Anotemos que esta participación porcentual varía según el tipo de material al cual hagamos referencia. Por ejemplo, si consideramos solamente los textos que son libros, la proporción de los autores ecuatorianos disminuye de un $25 \%$ a un $23 \%$. Esto era de esperarse, pues las referencias de documentos, leyes y periódicos son casi todas relativas al Ecuador.

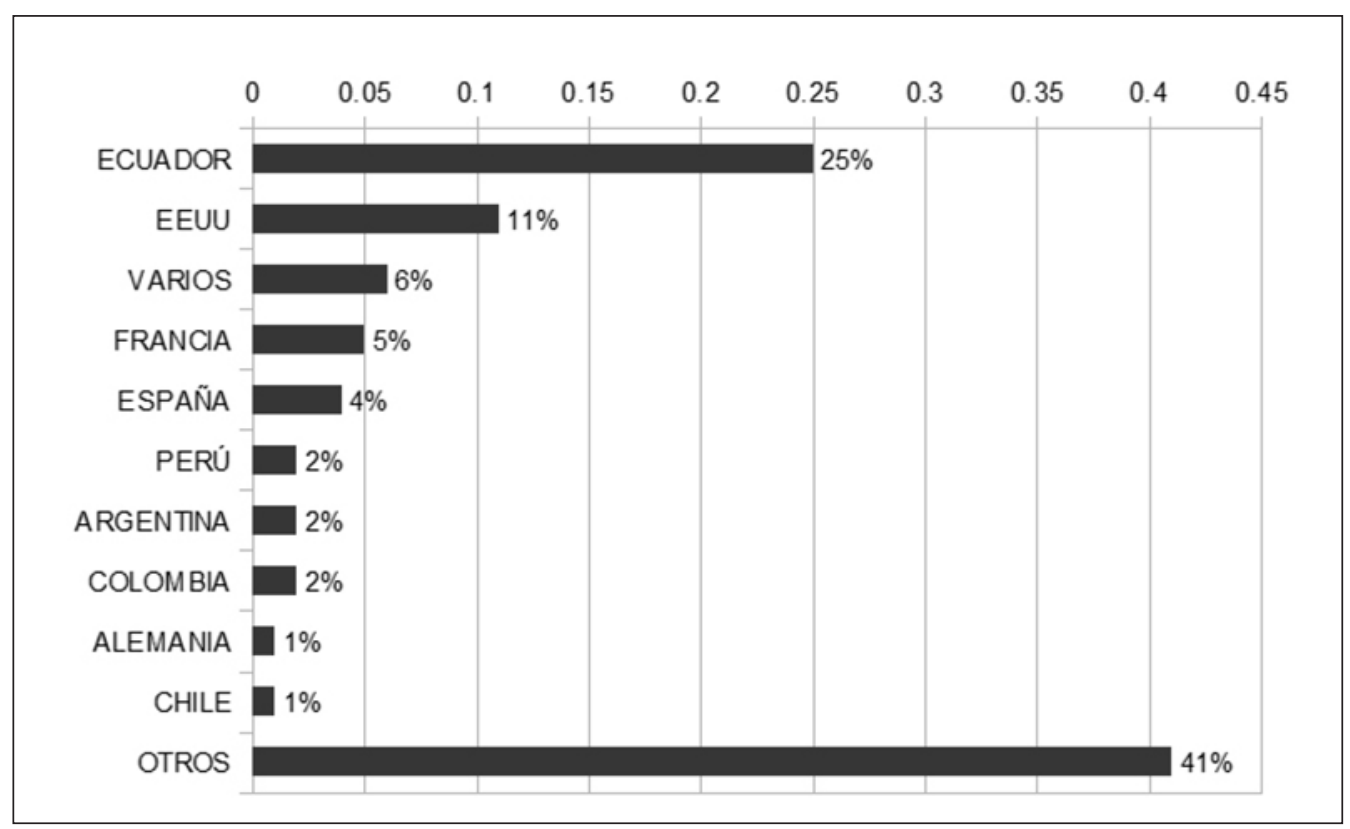

Gráfico 3. NaCionalidades de los AUtORES 
Esta reflexión que se relaciona con quienes se conecta esta actividad académica podría ser complementada con otra variable: el idioma en el cual están escritos los textos. Lógicamente, la gran mayoría son textos en castellano. En segundo lugar están los textos en inglés y muy atrás otros idiomas (francés, italiano, portugués) y en un nivel aún menor textos en lenguas indígenas.

\begin{tabular}{|l|c|}
\hline Cuadro 3. Tipo de entidades Que Publican los Materiales usados EN LaS tesis. Elaboración Propia \\
\hline Castellano & $84,80 \%$ \\
\hline Inglés & $14,14 \%$ \\
\hline Francés & $0,65 \%$ \\
\hline Portugués & $0,15 \%$ \\
\hline Italiano & $0,06 \%$ \\
\hline Quechua & $0,06 \%$ \\
\hline Shuar & $0,04 \%$ \\
\hline Hoao & $0,04 \%$ \\
\hline
\end{tabular}

Abordemos ahora la pregunta de cuán actual es el material utilizado. Para ello hemos construido un indicador que consiste en el tiempo promedio en años de antigüedad de los materiales citados al momento de la aprobación de la tesis. El valor que obtenemos es de 11,41 años. Esto nos da una idea respecto a cuan actuales son los materiales utilizados. ${ }^{5}$

Un último aspecto a abordar al tratar las características de la bibliografía son los temas de género. ¿De este total de referencias que proporción son mujeres? Dado que hay muchos autores institucionales y colectivos, donde no es posible asignar la autoría según criterios de género, hemos creado un indicador que es la proporción de mujeres sobre los hombres. Es decir, excluimos todos los colectivos o autores colectivos que incluyen hombres y mujeres. El resultado para este total es de 0,29. Este valor puede ser útil para establecer futuras comparaciones. Para comenzar señalemos que este valor sube a 0,35 en el caso de las tesis cuyos autores son mujeres y es de 0.206 para los autores de género masculino.

Otro elemento de análisis considerado dentro de las variables cuantitativas es la extensión de la tesis. El promedio de extensión es de 145,6 páginas, y la diferencia en extensión entre el pregrado y el nivel de maestría, no es mayor, pues las tesis de pregrado son en promedio un $8 \%$ más largas que las tesis de maestría. Si examinamos máximos y mínimos tenemos que en pregrado la extensión varía entre 77 y 301 páginas. En el postgrado esta variación va de 81 a 278 páginas. Se trata de valores curiosamente similares. ${ }^{6}$

Las posibilidades de desarrollar sobre esta base comparaciones a partir de sub agregados es muy grande. En esta primera aproximación nos limitaremos a explorar la dimensión temporal. Vamos a comparar las tesis aprobadas antes del año 2006, con aquellas que se terminaron desde esa fecha en adelante.

La primera pregunta se refiere a si la tesis está cambiando en cuanto a sus dimensiones. El dato que obtenemos es que las tesis anteriores al 2005, tenían un extensión promedio de 155 páginas y descienden a 133,6 . Hay otros datos que parecerían corroborar esta tendencia, por

5 Algunas aclaraciones respecto a este indicador. Se toma como referencia el año de publicación de la edición utilizada, por lo mismo un texto clásico de Marx, escrito en 1848, pero tomado de una edición de 1988, aparecer con esta última fecha. Por otra parte anotemos que evidentemente hay tesis en las cuales la utilización de documentos de la época investigada aumenta considerablemente este promedio.

6 En base a datos de las bibliotecas encontramos en Universidades de Quito tesis de pregrado con una extensión media de 134 páginas y de Maestría una extensión media de 128 páginas en este caso la muestra es de 112 tesis. 
ejemplo en otro universo de 99 tesis de maestría encontramos que la media de extensión pasa de 144 páginas a 118. ${ }^{7}$ Un dato complementario es la cantidad de referencias bibliográficas, que también disminuye de 80 a 70 , lo cual mantiene en 0,51 el número de referencias por página de tesis.

Para analizar el tipo de documentos utilizados en este marco temporal de realización de la tesis, hemos creido conveniente utilizar una nueva forma para resentar los resultados. En los siguiente gráficos de barras se ha consignado la diferencia entre la participación porcentual de cada categoría en el período posterior al 2006 con respecto al promedio total. Esto nos indica que tendencia de crecimiento o decrecimiento podemos detectar.

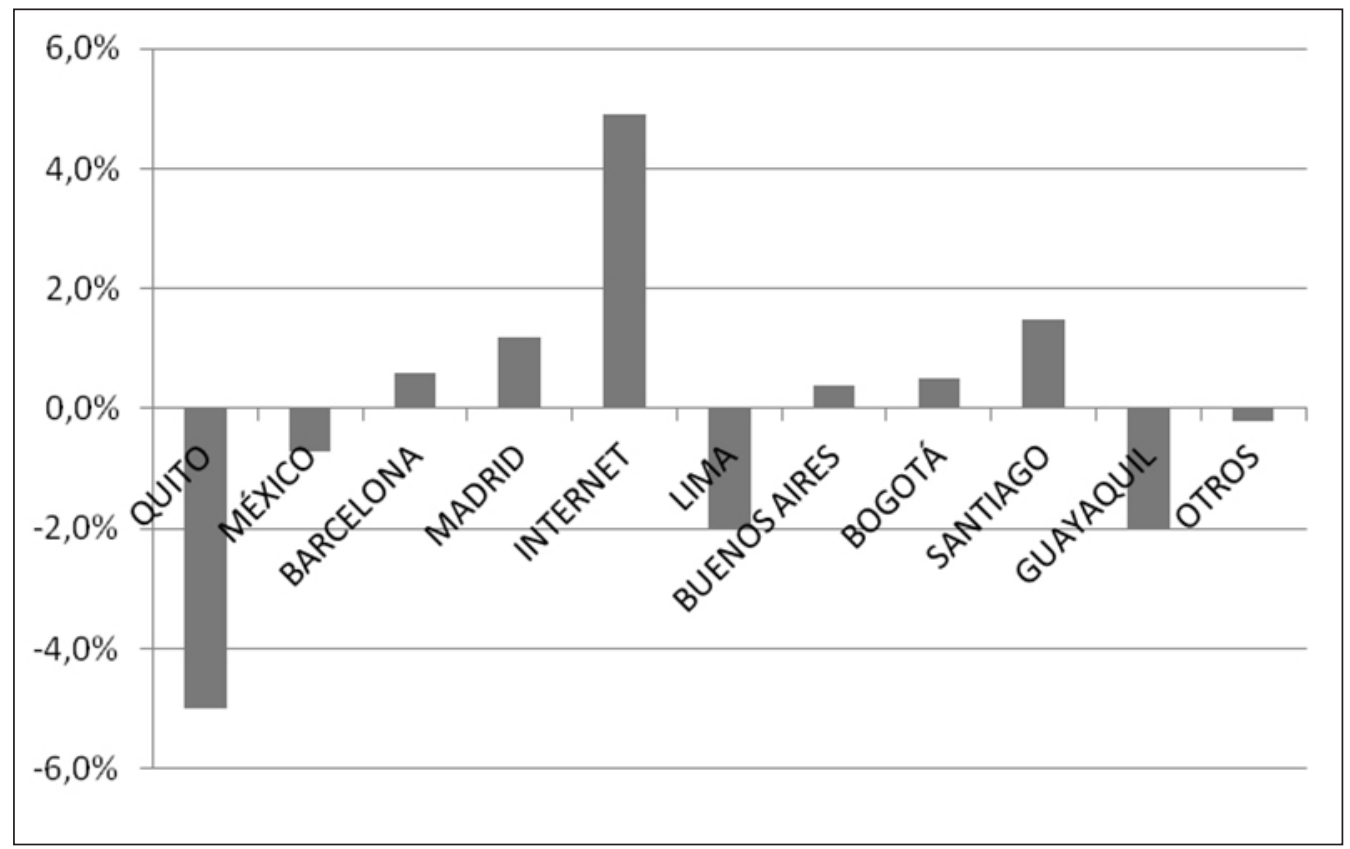

Gráfico 4. VARIACIONES PORCENTUALES de LOS DIVERSOS TIPOS DE DOCUMENTOS DE LAS TESIS POSTERIORES AL 2005 RESPECTO AL TOTAL. Elaboración PROPIA.

Como se puede ver, anteriormente se utilizaban más libros, artículos, textos manuscrito, y prensa. Ahora las referencias son más sobre capítulos de libros, internet y en alguna medida informaciones de conferencias.

Respecto a la ciudad de edición las tendencias muestran que hay ciudades que han ganado peso, y otras retroceden. La que pierde importancia de manera más decisiva es Quito, pues disminuye varios puntos porcentuales en su peso relativo. También lo hacen Lima y Guayaquil. Crecen en cambio, el internet, Madrid, Santiago, Barcelona y en menor medida Bogotá.

7 Son estimaciones preliminares, pues la cantidad de tesis en uno y otro período es muy desigual. 


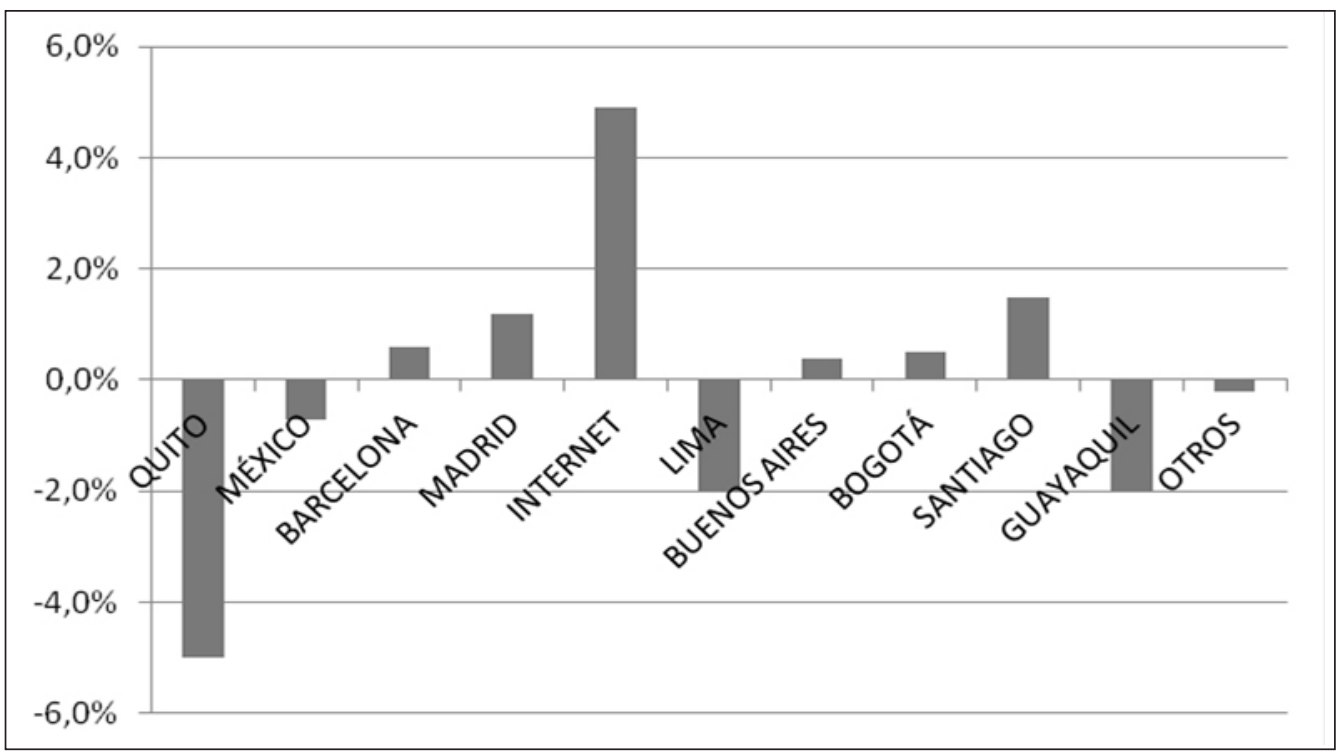

Gráfico 5. Variación de las diferentes CIUdAdes dónde han SIDO EDITAdos los DOCUMENTOS Utilizados en las tesis. Elaboración PROPIA.

En cuanto a las editoriales, la tendencia que observamos es un muy importante incremento de los textos editados por Flacso y de textos obtenidos de internet a los cuales no se les pudo asignar otra editorial. Disminuyen todos los demás.

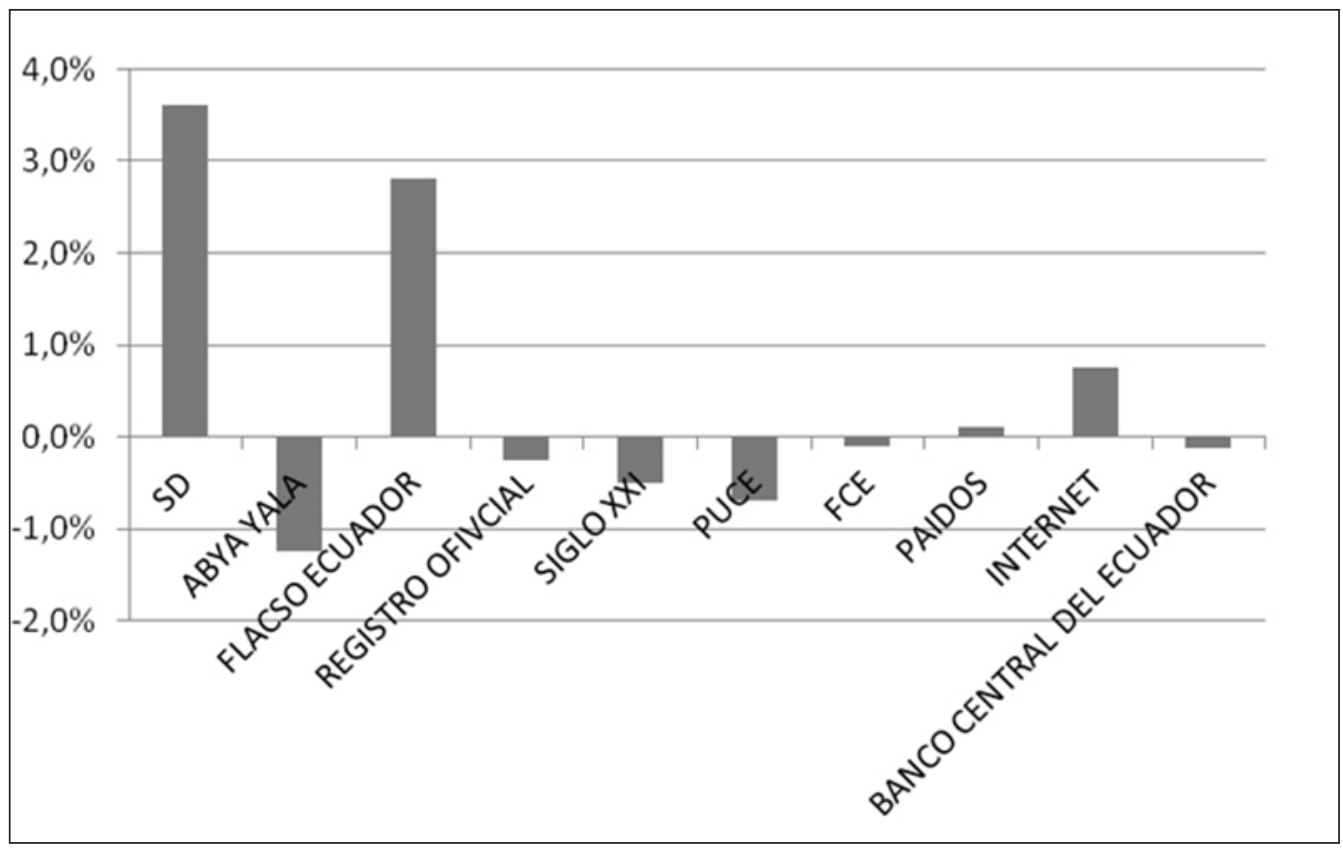

GrÁfico 6. VARIACión de LAS DIFERENTES EDITORIALES dóNDE HAN SIDO EDITADOS LOS DOCUMENTOS UTILIZADOS EN LAS TESIS. ElABORACIÓN PROPIA. 
Si procedemos a una clasificación de grandes grupos de entidades editoras vemos que el incremento fundamental se produce respecto a las editoriales universitarias, y también respecto al Internet. Todas las demás fuentes reducen su participación a excepción de los documentos producidos por entidades oficiales.

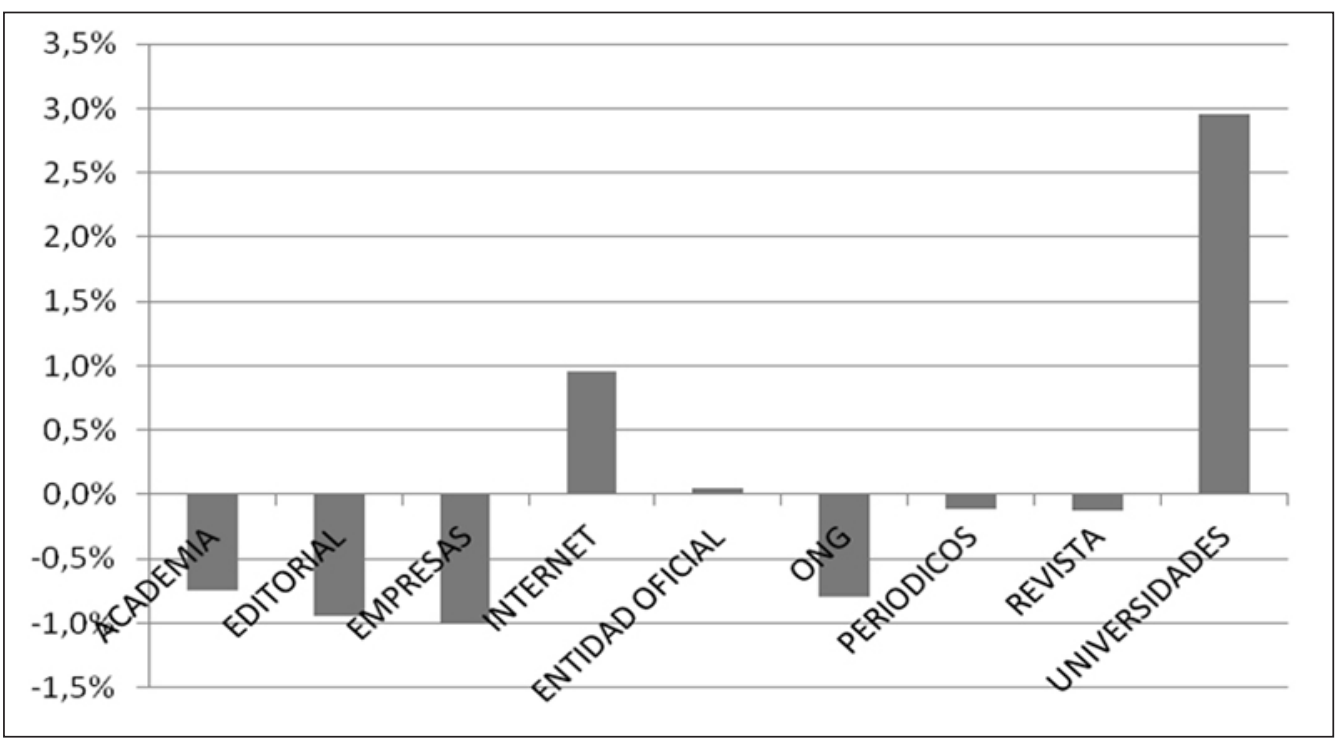

GrÁFICO 7. VARIACIÓN dE LAS dIFERENTES TIPOS DE ENTIDADES DÓNDE HAN SIDO EDITADOS LOS DOCUMENTOS UTILIZADOS EN LAS TESIS. ElaboraCión PROPIA.

En nuestra secuencia el siguiente punto ha sido la nacionalidad de los autores. Este es el gráfico de variaciones que obtenemos al comparar las tesis más recientes con el grupo total:

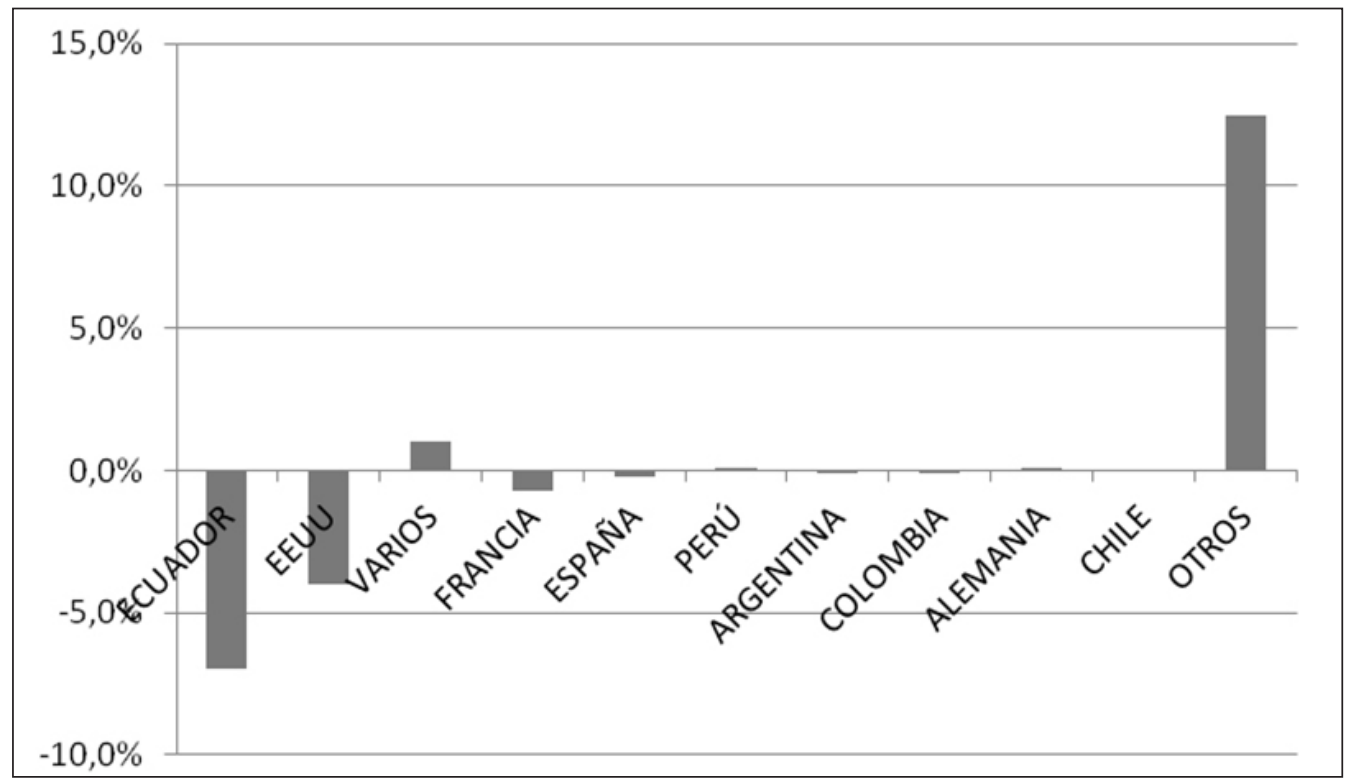

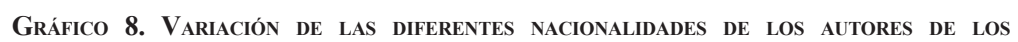
DOCUMENTOS UTILIZAdOS EN LAS TESIS. ElaboraCión PROPIA. 
Una disminución del peso porcentual de los autores ecuatorianos y norteamericanos y un aumento de dos grupos: los trabajos realizados por grupos que reúnen a autores de varias nacionalidades y de autores de otros países. Esta tendencia se mantiene con pocas variaciones si analizamos solamente los libros. En el gráfico 9 solamente hemos consignado las nacionalidades cuya participación más crece o decrece. En ella es llamativo el crecimiento de la presencia de libros de autores bolivianos.

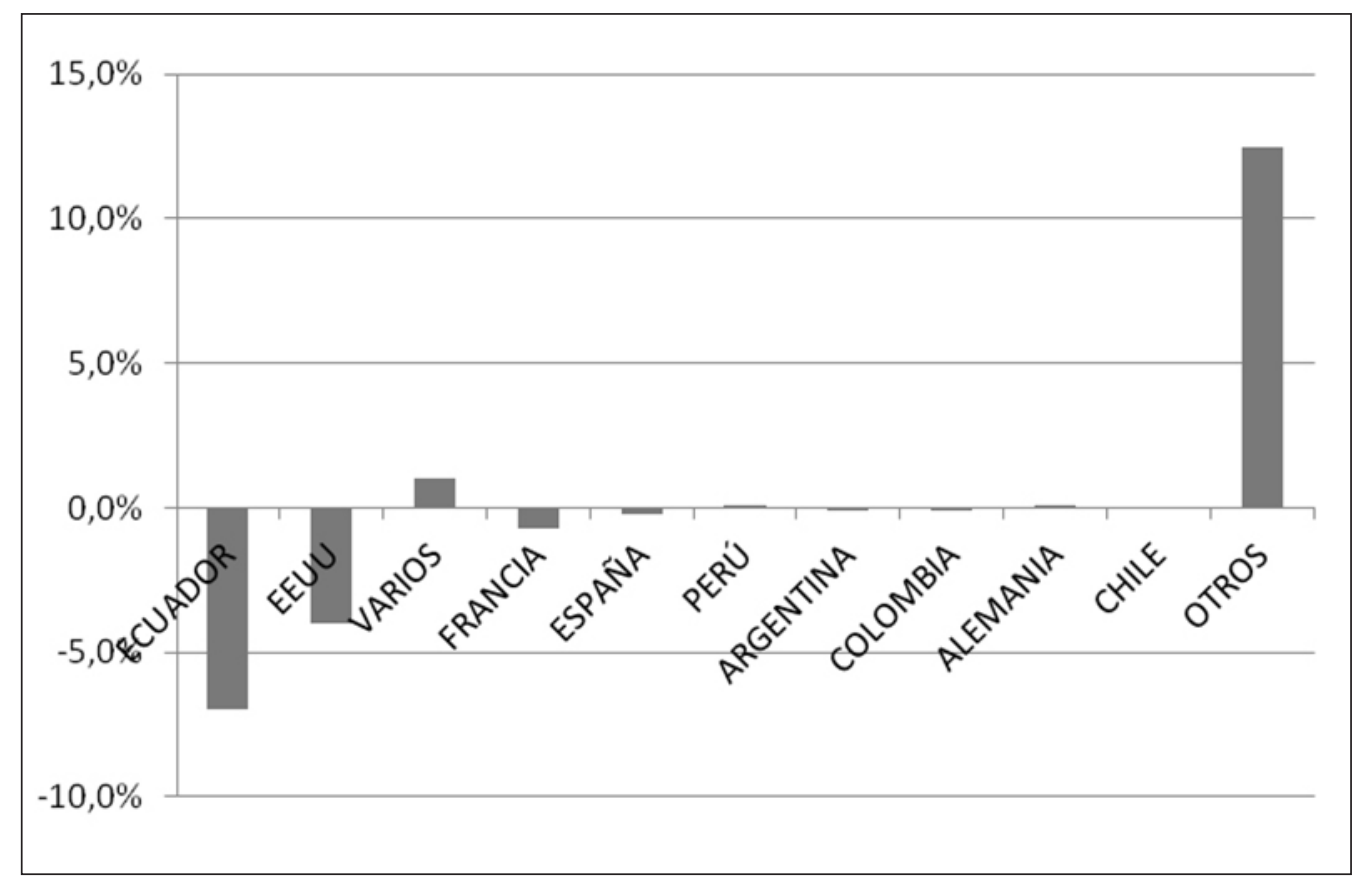

Gráfico 9. Variación de las diferentes nacionalidades de los aUtores de Libros UTILIZAdOS EN LAS TESIS. ElabORACIÓN PROPIA.

Abordando ahora el idioma usado, constatamos modificaciones que no son de gran importancia, pero se observa una disminución del uso de castellano del 0,45 \%. Entre los idiomas extranjeros solo crece en importancia el inglés, y lo hace en una proporción que casi llega al 1 $\%$. Todos los otros idiomas extranjeros decrecen y los idiomas indígenas mantienen una posición siempre muy marginal.

El último tema que hemos tratado es el relativo al papel de las mujeres como autoras. Señalemos algunos datos no esperados. La proporción de referencias de mujeres, tiene una muy ligera disminución. Pasa de 0,29 a 0,2818. Esta diferencia puede no ser significativa, pero si encontramos modificaciones más importantes si diferenciamos a los autores por sexo. En efecto entre las mujeres la participación de referencias de mujeres crece, aunque poco, pasa de 0,35 a 0369. Pero en cambio entre los varones encontramos una marcada disminución, se pasa de 0,206 a 0,134 . Esto nos indicaría un estancamiento de la participación de las mujeres como referente académico, y además una "guetización" del mismo, es decir una tendencia a que las mujeres se conviertan en un grupo diferenciado que escriben y se leen entre ellas, avanzando menos en la participación del mundo académico general.

La construcción de una base de datos de este estilo ofrece múltiples posibilidades, de anotar por ejemplo cómo evolucionan los autores más citados, de cómo diferentes escuelas, facultades y universidades se relacionan con espacios geográficos, o con corrientes teóricas. Sin embargo 


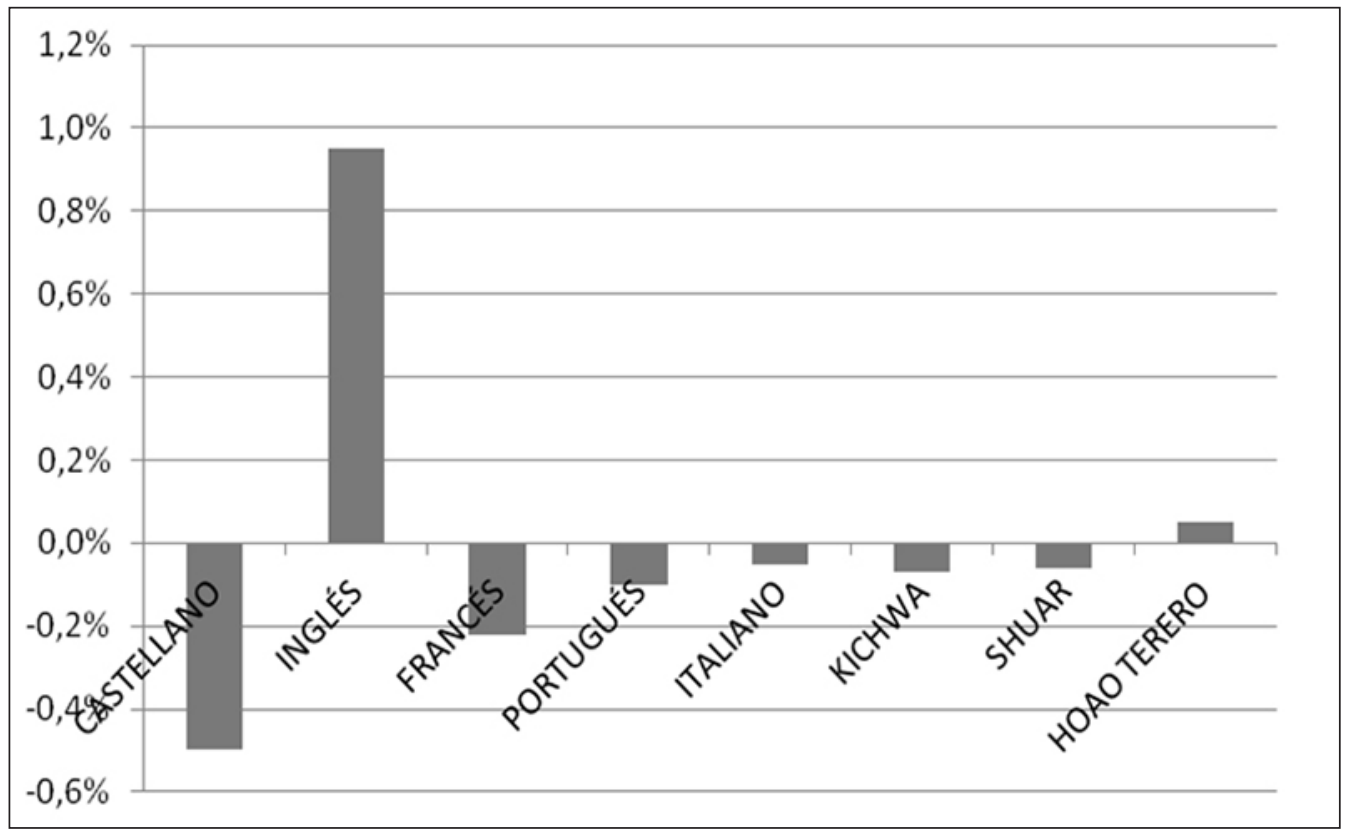

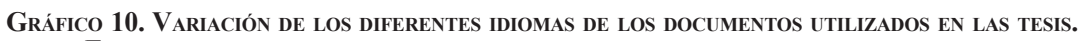
ELABORACIÓN PROPIA.

las debilidades de nuestro diseño muestral, nos recomiendan prudencia al momento de hacer sugerencias adicionales a las aquí formuladas.

A manera de miscelánea presentemos solamente algunos datos sobre los autores más citados, de los cinco más citados en este universo, tres son franceses, y dos ecuatorianos, mostrando con ello que los autores ecuatorianos individuales si tienen relevancia en las interlocuciones de esta actividad académica, y en el caso de los franceses, que como nacionalidad tienen menos peso que los norteamericanos, nos dice que son pocas figuras las que concentran gran parte de la atención.

Podemos agregar una última comparación adicional. Si nos preguntamos cuanto varían los autores y los textos utilizados en el tiempo, obtenemos un resultado que a nosotros nos llama la atención, y este es que entre estos dos períodos, solamente el 5,2\% de las referencias bibliográficas resultan comunes. Esta comparación puede expresarse a través de un índice estadístico estandarizado como es el Índice de Jacard, que de todas maneras es muy bajo 2,7\%. Lo que nos estaría indicando que casi todo el marco de referencias ha sido cambiado y modificado en este período.

\section{Comentarios}

El material que hemos expuesto nos lleva a formularnos una serie de preguntas. Son hipótesis insinuadas por un material que tiene una representatividad acotada, y que por lo tanto requerirían de verificaciones ulteriores, pero que de todas maneras pueden ser útiles. En primer lugar surge la interrogante sobre ¿Qué son las tesis? Qué papel cumplen. Los materiales aquí trabajados insinúan que son esfuerzos académicos no despreciables. 130 páginas representan un esfuerzo que no puede ser desvalorizado, y 80 o 70 referencias parecen indicarnos un esfuerzo apreciable de interacción intelectual. Esto a pesar de que la experiencia de este trabajo nos muestra que las bibliografías en las tesis no son elaboradas con demasiada rigurosidad. Hay mucho errores y muy frecuentes. Desde referencias con datos incompletos hasta referencias repetidas, es decir aparece 
el mismo texto una vez como obra de un autor institucional y otra como producto de un autor individual. Esto para no mencionar la marcada carencia de estandarización, y esto especialmente en relación a los textos legales.

Surgen también preguntas, por ejemplo cuál es la diferencia entre las tesis de pregrado y las de postgrado. La extensión no es un factor claro, tampoco es la intensidad de consulta con fuentes. La que aparece con claridad como una diferencia sustancial en cuanto a monto de trabajo implicado es la que existe entre tesis de doctorado y todas las demás. Pero aún esto es relativo, pues la propia clasificación de pre-grado, puede ser objeto de controversia, así tenemos por ejemplo que las tesis de doctor en leyes, que hemos identificado se parecen claramente a las tesis doctorales de postgrado. En todo caso la propuesta implícita en esta inicial prospección es que para tratarlo es útil y relevante no sólo mirar los modelos que pudiéramos proponernos sino lo que ha sido la realidad y la práctica en el país al respecto.

La segunda gran interrogante que puede ser suscitada a partir de estos materiales, es si es que nos sirven para discutir, dos preguntas relacionadas y en alguna medida contrapuestas. La primera es sí existe un espacio de discusión académica en el Ecuador. Quienes hacen tesis usan, siguen y refutan a personas que escriben en este país. Pero esto tiene también matices, la evolución que percibimos es la de una fuerte agudización de un quito-centrismo académico, que da que pensar, no sólo por el desequilibrio señalado, sino porque este parece agudizarse con el tiempo.

En cuanto a la conexión con el mundo académico global aparecen algunos resultados interesantes. Pierden importancia ciudades que anteriormente fueron referentes intelectuales importantes para el Ecuador, nos referimos a Lima y México, y ganan sobre todo dos ciudades en las cuales hay una intensa actividad de traducción al castellano de libros de muchos países. Nos referimos a Barcelona y Madrid. Pensábamos que un rol similar podrían haber tenido ciudades como Bogotá y Buenos Aires. Nustros datos nos confirman esto.

$\mathrm{Y}$ esto nos lleva a otra cosa, de todas maneras el porcentaje de textos editados en Quito es sustancialmente mayor al de autores ecuatorianos, lo cual nos indica que ha existido un esfuerzo apreciable de traducción y difusión. Es claro que el eje de este esfuerzo ha sido la editorial Abya Yala, la misma que tiene un rol destacado como fuente de referencia para los tesistas en Ciencias Sociales.

Cabe señalar sin embargo que este rol parece haber sido parcialmente desplazado por otro fenómeno y este es el de una producción y también de un acceso más importante a editoriales universitarias que en están asumiendo un rol creciente. En los casos por nosotros abordados habría unA editorial universitaria en el Ecuador que participa de esta tendencia, la Flacso. Este tema nos lleva a otro y este es el de la valoración de las publicaciones y su reconocimiento como "académicas" Este es un proceso que se ha llevado a cabo fundamentalmente en centros metropolitanos, y lleva a ciertas paradojas. Sino consideramos a la editorial Abya Yala como una editorial académica, estamos ignorando un importante y sostenido esfuerzo realizado durante muchos años en el país. Estamos creando una discriminación en contra de quienes tienen menos acceso a las formas de publicación del Norte.

Si nos ocupamos del nivel de actualidad de los textos utilizados. Nos encontramos que prácticamente no existen cambios. La edad promedio de las referencias es de alrededor de 11 años. Y esto permanece constante en el tiempo. Llama la atención sin embargo lo que parecería ser un indicador de una alta tasa de renovación. Aparentemente en cada década se cambian los referentes, Esto nos puede indicar un alto nivel de renovación, o tal vez una ruptura de continuidad en los marcos que se utilizan y del proceso reflexivo mismo. Sin embargo por ahora estos son sólo indicios. Se requieren de técnicas de muestreo diferentes para sacar conclusiones más claras.

Pero regresemos a la discusión sobre el eventual "parroquialismo" de la producción académica. Otro indicador de esto, es la utilización de idiomas. Constatamos que la bibliografía en idioma extranjero es relativamente reducida, mucho menor a la utilización de textos producidos originalmente en otros idiomas. Hay una ligera tendencia al incremento del uso del inglés, pero parece claro que por algún tiempo más predominara masivamente el uso del idioma castellano para acceder a los materiales que necesitan los tesistas. A partir de esto podemos plantearnos 
algunas otras cuestiones. Una concepción de la apertura al universalismo de la Ciencia postularía que esta no debe conformarse con el acceso al idioma de la globalización, sino que debe proponerse estar en condiciones de alimentarse de textos en diversos idiomas. Una pregunta específica puede surgir respecto al portugués, idioma cercano lingüística y geográficamente, pero que no parece tener mayor incidencia en las tesis hasta el momento. También nos ha llamado la atención la ausencia total de textos en ruso y en alemán. En el primer caso se trató de una legua que decenas de ecuatoriano aprendieron a través de las becas que la Unión Soviética ofreció durante muchos años. No parece haber quedado huella de ello. El caso del alemán, que es el idioma de un conjunto apreciable de autores utilizados por los tesistas, y que cuenta con vínculos de cooperación, becas e intercambio importantes con el Ecuador tampoco aparece.

Creemos que estos resultados llaman la atención sobre el valor y la importancia de dos aspectos. Consideramos que los esfuerzos por dotar a los estudiantes de acceso a otras lenguas deben ir más allá de la enorme concentración actual en el inglés. El portugués tiene algunas razones geográficas y temáticas que podrían sustentar una mayor atención. El caso de francés en retroceso pero con cierta tradición presente también debe ser considerado. Es también algo claro la marginalidad de los idiomas indígenas a pesar de que entre las tesis identificadas existen temáticas directamente relacionadas con los pueblos indígenas.

Dentro de esta misma temática podemos regresar a la perspectiva del rol de la traducción. Esta actividad ha sido generalmente subvalorada, creemos que es pertinente defenderla, no hay quien asimile mejor un conjunto de ideas que el que hace un esfuerzo de traducción de ellas. Se trata además de un esfuerzo necesario. Nuestra opinión es que esta tarea no debe ser subvalorada $\mathrm{y}$ al contrario debe tener un importante reconocimiento.

Un último aspecto a considerar es el relativo a la participación de mujeres. Nos ha llamado la atención los resultados relativamente modestos de la utilización de textos escritos por mujeres. Con seguridad el problema de que este sondeo no diseño una estrategia para incorporar explícitamente la dinámica de la actividad académica en torno a género, puede ser una explicación. Pero de todas maneras llama la atención la creciente diferencia en la utilización de acceso a textos de hombres y mujeres entre estos dos subgrupos. Hay síntomas de un enclaustramiento de las académicas mujeres en temas de género, que podría llevar a un mundo académico femenino desarticulado del masculino que todavía es el mayoritario.

Por último abordemos la pregunta de qué se debe hacer a futuro. Tomemos en cuanta que una persona con cierto entrenamiento ya en el uso de la base de datos requiere entre 4 y 5 horas para procesar la bibliografía de una tesis promedio a las cuales cabría agregar un tiempo no despreciable para el diseño de las diversas formas de analizar el material y los resultados. Sin embargo hacerlo puede ser algo útil para reflexionar sistemáticamente sobre lo que es esta actividad tan importante para la educación superior del país.

\section{Bibliografía:}

Arcos Carlos. 2012, ¡No es un jardín de infantes! Reflexiones en torno al Proyecto de Reglamento de carrera $Y$ escalafón de Profesor e investigador del sistema de educación Superior, Documento de Trabajo, Instituto de Altos Estudios Nacionales, Quito.

FLACSO, sede Ecuador Biblioteca. 2014, Base de datos biblioteca https://www. flacso. edu.ec/portal/paginas/ biblioteca.16 (último acceso 14 dic 2014).

De Sousa Santos Boaventura. 2005, La Universidad en el Siglo XXI, para un reforma democrática y emancipadora de la Universidad, Centro de Investigaciones Interdisciplinarias en Ciencias y Humanidades UNAM, México.

Pontificia Universidad Católica del Ecuador, Biblioteca. 2014, http://www.puce. edu.ec/sitios/biblioteca/ (último acceso diciembre 2014).

Universidad San francisco de Quito, Biblioteca http://repositorio.usfq.edu.ec/ (último acceso diciembre 2014).

Velasco Fernando. 1972, "Ecuador Subdesarrollo y Dependencia”, Tesis de Economía PUCE, Quito.

Villavicencio Arturo. 2013, ¿Hacia dónde va el proyecto Universitario de la Revolución Ciudadana?, 13 ediciones, Quito. 